\title{
SGLT2 inhibitors in non-diabetic kidney disease
}

\author{
Vladimír Tesař ${ }^{C-F}$ \\ Department of Nephrology, Charles University in Prague, Czech Republic \\ $A$ - research concept and design; $B$ - collection and/or assembly of data; $C$ - data analysis and interpretation; \\ $D$ - writing the article; $E$ - critical revision of the article; $F$ - final approval of the article
}

\section{Address for correspondence}

Vladimír Tesar̆

E-mail: vladimir.tesar@vfn.cz

\section{Funding sources \\ None declared}

Conflict of interest

None declared

Acknowledgements

Research initiative of the General University Hospital. Grant No. DRO VFN 64165 from the Ministry of Health of the Czech Republic.

Received on December 5, 2021

Accepted on January 11, 2022

Published online on January 25, 2022

Cite as

Tesař V. SGLT2 inhibitors in non-diabetic kidney disease.

Adv Clin Exp Med. 2022;31(2):105-107.

doi:10.17219/acem/145734

DOI

10.17219/acem/145734

Copyright

Copyright by Author(s)

This is an article distributed under the terms of the

Creative Commons Attribution 3.0 Unported (CC BY 3.0)

(https://creativecommons.org/licenses/by/3.0/)

\begin{abstract}
There is an accumulating evidence demonstrating renoprotective and cardioprotective role of sodiumglucose cotransporter 2 (SGLT2) inhibitors in early to advanced diabetic kidney disease. Data from recently published Dapagliflozin and Prevention of Adverse Outcomes in the Chronic Kidney Disease (DAPA-CKD) trial clearly show that dapagliflozin is similarly renoprotective in non-diabetic chronic kidney disease in a wide range of estimated glomerular filtration rate (eGFR) of $25-75 \mathrm{~mL} / \mathrm{min} / 1.73 \mathrm{~m}^{2}\left(0.42-1.25 \mathrm{~mL} / \mathrm{s} / 1.73 \mathrm{~m}^{2}\right)$ and albumin/creatinine ratio 200-5000 mg/g (approx. 20-500 mg/mmol). Patients with type 1 diabetes, autosomal dominant polycystic kidney disease, antineutrophil cytoplasmic antibody (ANCA)-associated vasculitis and lupus nephritis were excluded from the study, but, on the other hand, prespecified subanalysis demonstrated that dapagliflozin should be renoprotective also in patients with immunoglobulin A (IgA) nephropathy. The renoprotective effect of SGLT2 inhibitors is additive to the renoprotection conferred with blockers of renin-angiotensin system, including both inhibitors of angiotensin converting enzyme (ACEI), or angiotensin receptor blocker (ARB). These promising data will be hopefully confirmed by the ongoing the Study of Heart and Kidney Protection With Empagliflozin (EMPA-KIDNEY) trial, the results of which are expected later in 2022.
\end{abstract}

Key words: SGLT2 inhibitor, dapagliflozin, chronic kidney disease 


\section{Introduction}

Inhibitors of sodium-glucose cotransporter 2 (SGLT2) in the proximal tubule of the kidney were shown to slow down the progression of diabetic kidney disease (DKD) and to lower in patients with DKD the cardiovascular morbidity and mortality.

Dapagliflozin, the SGLT2 inhibitor tested in the Dapagliflozin and Prevention of Adverse Outcomes in the Chronic Kidney Disease (DAPA-CKD) study, ${ }^{1}$ was already demonstrated to reduce renal risk in the Dapagliflozin Effect on CardiovascuLAR Events (DECLARETIMI) study with primary cardiovascular endpoints in patients with low renal risk. ${ }^{2}$ Renoprotective effect of another SGLT2 inhibitor - canagliflozin - was recently demonstrated in the Canagliflozin and Renal Events in Diabetes with Established Nephropathy Clinical Evaluation (CREDENCE) study with primary renal endpoints in patients with type 2 diabetes and chronic kidney disease (CKD) with high renal risk. ${ }^{3}$ As SGLT2 inhibitors do not induce hypoglycemia in non-diabetic patients and their renoprotective effect seems to be predominantly hemodynamically mediated (decrease of glomerular pressure due to constriction of the pathologically dilated afferent arteriole), they were supposed to be renoprotective also in non-diabetic patients.

\section{The DAPA-CKD study}

The DAPA-CKD study recruited 4304 patients with CKD and either type 2 diabetes or without type 2 diabetes, with estimated glomerular filtration rate (eGFR) of $25-75 \mathrm{~mL} / \mathrm{min} / 1.73 \mathrm{~m}^{2}\left(0.42-1.25 \mathrm{~mL} / \mathrm{s} / 1.73 \mathrm{~m}^{2}\right)$ and albumin/creatinine ratio of $200-5000 \mathrm{mg} / \mathrm{g}$ (approx. $20-500 \mathrm{mg} / \mathrm{mmol}$ ) on stable dose of either inhibitor of angiotensin converting enzyme (ACEI) or angiotensin receptor blocker (ARB), or not tolerating any of them. Patients were randomized in a ratio 1:1 to either SGLT2 inhibitor dapagliflozin (at a dose of $10 \mathrm{mg}$ daily) or placebo. Patients with type 1 diabetes, autosomal dominant polycystic kidney disease, antineutrophil cytoplasmic antibody (ANCA)-associated vasculitis, and lupus nephritis were excluded from the study. Mean age of recruited patients was $61.8 \pm 12.1$ years, mean eGFR was $43.1 \pm 12.4 \mathrm{~mL} / \mathrm{min} / 1.73 \mathrm{~m}^{2}$ and mean albumin/creatinine ratio was $949 \mathrm{mg} / \mathrm{g}$. Patients with diabetes represented $67.5 \%$ of patients. The study was preliminarily stopped after 2.4 years because of a clearly positive result.

Primary endpoint defined as the first occurrence of either decrease of GFR by $50 \%$, end-stage renal disease or cardiovascular or renal death occurred in $9.2 \%$ of patients treated with dapagliflozin and $14.5 \%$ of patients in the placebo limb (relative risk reduction 39\%, $\mathrm{p}<0.001)$. Dapagliflozin reduced the risk of all individual components of the primary endpoint, and the effect was consistent in all predefined subgroups. The risk of primary endpoint was reduced by $36 \%$ in patients with type 2 diabetes and by $50 \%$ in non-diabetic patients. Dapagliflozin also decreased the risk of composite renal endpoint by $44 \%$ and composite cardiovascular endpoint (cardiovascular mortality or hospitalization for heart failure) by $29 \%$. All-cause mortality was decreased by dapagliflozin by $31 \%$.

Dapagliflozin was very well tolerated and the occurrence of serious adverse events was similar in patients treated with dapagliflozin and placebo. There was no reported case of diabetic ketoacidosis in patients with type 2 diabetes and no severe hypoglycemia in non-diabetic patients with CKD.

The lower limit of eGFR in the CREDENCE study was $30 \mathrm{~mL} / \mathrm{min} / 1.73 \mathrm{~m}^{2}$. In the DAPA-CKD study, $14.5 \%$ of patients had eGFR lower than $30 \mathrm{~mL} / \mathrm{min} / 1.73 \mathrm{~m}^{2}$, so the DAPA-CKD study provided first data on the renoprotectivity of SGLT2 inhibitors in this population of patients with CKD and low eGFR. Moreover, the renoprotective (and cardioprotective) effect of SGLT2 inhibitors was extended from patients with type 2 diabetes to non-diabetic patients with CKD.

Importantly, the renoprotective effect of dapagliflozin observed in the DAPA-CKD study was consistent across the whole spectrum of recruited patients: it was comparable in patients with albumin/creatinine ratio higher and lower than $1000 \mathrm{mg} / \mathrm{kg}$ and in patients with eGFR both higher and lower than $45 \mathrm{~mL} / \mathrm{min} / 1.73 \mathrm{~m}^{2}$, although the patients with higher albuminuria and lower eGFR may benefit most. ${ }^{4}$ In a prespecified analysis, the effect of dapagliflozin on the rate of loss of eGFR was more expressed in patients with type 2 diabetes compared to non-diabetic patients, and in patients with higher glycated hemoglobin and albuminuria. ${ }^{5}$ In an interesting subanalysis, in 270 patients with immunoglobulin A (IgA) nephropathy recruited to the DAPA-CKD study, dapagliflozin reduced the primary outcome by $71 \%$, suggesting that dapagliflozin could have a great potential to improve renal outcome in the patients with most frequent glomerulonephritis. ${ }^{6}$

\section{Other recent and ongoing studies in DKD and non-diabetic CKD}

There is a great unmet need in non-diabetic CKD patients. In patients with type 2 diabetes, we currently have the evidence for renoprotective effect of not only the inhibition of the renin-angiotensin system (e.g. The Irbesartan Diabetic Nephropathy Trial (IDNT) study ${ }^{7}$ ), but also endothelin type A (ETA) receptor antagonist atrasentan (Study Of Diabetic Nephropathy With Atrasentan (SONAR) study $^{8}$ ) and non-steroidal selective inhibitor of mineralocorticoid receptor finerenone (FIDELIO-DKD study ${ }^{9}$ ). 
In non-diabetic patients with CKD, there is an evidence for renoprotective effect of the renin-angiotensin system (RAS) blockade only. ${ }^{10}$ It is important to stress than in the DAPA-CKD study, most patients were treated with RAS inhibitors, so the renoprotective effect of dapagliflozin is additive to the effect of the RAS blockade.

Since patients with CKD (and especially patients with DKD) have high cardiovascular morbidity and mortality, any renoprotective drug should be also cardioprotective, or at least without increased cardiovascular risk. Some previous studies in patients with DKD were prematurely discontinued, e.g., studies with sequential inhibition of the RAS either with the combination of lisinopril and losartan ${ }^{11}$ or aliskiren and losartan, ${ }^{12}$ because of an increased rate of cardiovascular events partly related to hyperkalemia. It is important to emphasize that in the DAPACKD study, dapaglifozin also decreased all-cause mortality and cardiovascular morbidity (hospitalization for heart failure and mortality).

Based on the data from CREDENCE and DAPA-CKD studies, the SGLT2 inhibitors should be used in all patients with DKD and eGFR > 30 (or even 25) $\mathrm{mL} / \mathrm{min} / 1.73 \mathrm{~m}^{2}{ }^{13}$ Main contribution of DAPA-CKD is, however, the evidence for renoprotective effect of dapagliflozin also in patients with non-diabetic CKD, as for these patients for the last 20 years the only option is the inhibition of the renin-angiotensin system. Therefore, the results of the DAPA-CKD study are of utmost importance for all patients with CKD. Similar study, the Study of Heart and Kidney Protection With Empagliflozin (EMPA-KIDNEY), recruiting patients with type 2 diabetes and CKD and non-diabetic patients with CKD to either empagliflozin or placebo group, is currently ongoing and the results should be available later in $2022 .{ }^{13}$

Hopefully, based on the data from the DAPA-CKD study, the treatment with dapagliflozin should be also available for non-diabetic patients with CKD at risk of progression to end-stage kidney disease.

\section{ORCID iDs}

Vladimír Tesař (1) https://orcid.org/0000-0001-6982-0689

\section{References}

1. Heerspink HJL, Stefansson BV, Correa-Rotter R, et al; DAPA-CKD Trial Committees and Investigators. Dapagliflozin in patients with chronic kidney disease. N Engl J Med. 2020;383(15):1436-1446. doi:10.1056/ NEJMoa2024816

2. Wiviott SD, Raz I, Bonaca MP, et al; DECLARE-TIMI 58 Investigators. Dapagliflozin and cardiovascular outcomes in type diabetes. N Engl J Med. 2019;380(4):347-357. doi:10.1056/NEJMoa1812389

3. Perkovic V, Jardine MJ, Neal B, et al; CREDENCE Trial Investigators. Canagliflozin and renal outcomes in type 2 diabetes and nephropathy. N Engl J Med. 2019;380(24):2295-2306. doi:10.1056/NEJMoa1811744

4. Neuen BL, Jardine MJ, Perkovic V. Sodium-glucose cotransporter 2 inhibition: Which patient with chronic kidney disease should be treated in the future? Nephrol Dial Transplant. 2020;35(Suppl 1):i48-i55. doi:10.1093/ndt/gfz252

5. Heerspink HJL, Jongs N, Chertow GM, et al; DAPA-CKD Trial Committees and Investigators. Effect of dapagliflozin on the rate of declline in kidney function in patients with chronic kidney disease with and without type 2 diabetes: A prespecified analysis from the DAPACKD trial. Lancet Diabetes Endocrinol. 2021;9(11):743-754. doi:10.1016/ S2213-8587(20)30369-7

6. Wheeler D, Toto RD, Stefánsson BV, et al; DAPA-CKD Trial Committees and Investigators. A pre-specified analysis of the DAPA-CKD trial demonstrates the effects of dapagliflozin on major adverse kidney events in patients with IgA nephropathy. Kidney Int. 2021;100(1): 215-224. doi:10.1016/j.kint.2021.03.033

7. Parving $\mathrm{HH}$, Lehnert $\mathrm{H}$, Bröchner-Mortensen J, Gomis R, Andersen S, Arner P; Irbesartan in Patients with Type 2 Diabetes and Microalbuminuria Study Group. The effect of irbesartan on the development of diabetic nephropathy in patients with type 2 diabetes. NEngl J Med. 2001;345(12):870-878. doi:10.1056/NEJMoa011489

8. Heerspink HJL, Parving $\mathrm{HH}$, Andress DL, et al; SONAR Committees and Investigators. Atrasentan and renal events in patients with type 2 diabetes and chronic kidney disease (SONAR): A double-blind, randomised, placebo-controlled trial. Lancet. 2019;393(10184):1937-1947. doi:10.1016/S0140-6736(19)30772-X

9. Bakris GL, Agarwal R, Anker SD, et al; FIDELIO-DKD Investigators. Effect of finerenone on chronic kidney disease outcomes in type 2 diabetes. N Engl J Med. 2020;383(23):2219-2229. doi:10.1056/NEJ Moa2025845

10. The GISEN Group. Randomised placebo-controlled trial of effect of ramipril on decline in glomerular filtration rate and risk of terminal renal failure in proteinuric, non-diabetic nephropathy. Lancet. 1997;349(9069):1857-1863. PMID:9217756.

11. Fried LF, Emanuele $\mathrm{N}$, Zhang JH. Combined angiotensin inhibition for the treatment of diabetic nephropathy. NEngl J Med. 2013;369(8): 1892-1903. doi:10.1056/NEJMoa1303154

12. Parving $\mathrm{HH}$, Brenner BM, McMurray JJ, et al; ALTITUDE Investigators. Cardiorenal endpoints in a trial of aliskiren for type 2 diabetes. N Engl J Med. 2012;367(23):2204-2213. doi:10.1056/NEJMoa1208799

13. Herrington WG, Preiss $D$, Haynes $R$, et al. The potential for improving cardio-renal outcomes by sodium-glucose co-transporter-2 inhibition in people with chronic kidney disease: A rationale for the EMPAKIDNEY. Clin Kidney J. 2018;11(6):749-761. doi:10.1093/ckj/sfy090

14. KDIGO Diabetes Work Group: KDIGO 2020 Clinical Practice Guideline for Diabetes Management in Chronic Kidney Disease. Kidney Int. 2020;98(4S):S1-S115. doi:10.1016/j.kint.2020.06.019 